\title{
SUR LA PRÉSENCE DE PHLEBOTOMUS PAPATASI \\ SCOPOLI 1786 A MARSEILLE
}

\author{
Par J. RAYNAL et P. LE GAC
}

Le 15 juillet au matin, l'un de nous, habitant sur la Corniche, à Marseille, capturait dans sa moustiquaire deux phlébotomes, un mâle et une femelle.

L'étude de ces deux exemplaires nous permit de faire la détermination de deux Phlebotomus papatasi Scopoli 1786, d'après les principales caractéristiques suivantes :

$$
\text { MALE }
$$

Gouleur : jaune pâle.

\section{Dimensions}

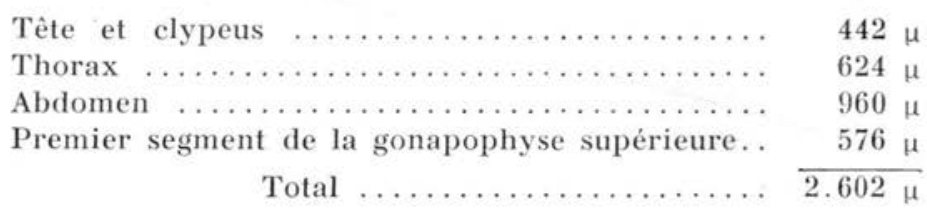

\section{Antennes}

$$
\begin{aligned}
& \mathrm{III}=\mathrm{IV}+\mathrm{V} . \\
& \mathrm{III}<\mathrm{IV}+\mathrm{V}+\mathrm{VI} . \\
& \mathrm{III}<\mathrm{XII} \ldots \mathrm{XVI} . \\
& \mathrm{IV}+\mathrm{V}<\mathrm{XII} \ldots \mathrm{XVI} . \\
& \mathrm{IV}+\mathrm{V}+\mathrm{VI}>\mathrm{XII} \ldots \mathrm{XVI} .
\end{aligned}
$$

Les épines géniculées, petites et courtes, sont bigéminées. La formule antennaire est done :

$$
\frac{2}{\text { III }-\mathrm{XV}}
$$

La formule palpale est : $1,4,2,3,5$.

$$
\begin{array}{ll}
\mathrm{II}<\mathrm{III} . & \\
\mathrm{V}=\mathrm{II}+\mathrm{III} . & \mathrm{V} \\
\mathrm{V} & >\mathrm{III}+\mathrm{IV} . \\
\mathrm{V} & <\mathrm{II}+\mathrm{III}+\mathrm{IV} .
\end{array}
$$

Anvales de Parasitologie, T. $\mathrm{X}, \mathrm{N}^{\circ} 6 .-1^{\text {er }}$ novembre 1932 , p. 497-503, 33. 


\section{Epipharynx}

La longueur de l'épipharynx est de $28.5 \mu$.

Le rapport :

$$
\frac{\text { palpe }}{\text { épipharynx }}=3,09
$$

\section{Aile}

Le bord postérieur de l'aile est légèrement plus arqué que le bord antérieur. La première nervure longitudinale recouvre la branche antérieure de la deuxième sur le quart de sa longueur.

La branche antérieure de la deuxième nervure longitudinale est plus longue que la distance entre les deux fourches : $\alpha>\beta$.

La fourche postérieure de la deuxième nervure longitudinale est plus rapprochée de la base de l'aile que la fourche de la quatrième.

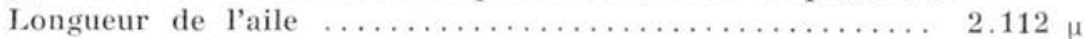

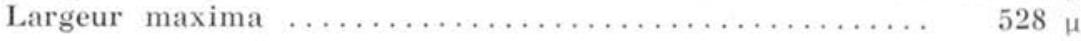

$$
\begin{aligned}
& \frac{\text { Longueur de l'aile }}{\text { Largeur de l'aile }}=4 \\
& \frac{\text { Taille de l'insecte }}{\text { Longueur de l'aile }}=1,26
\end{aligned}
$$

$$
\begin{aligned}
& \alpha=365 \mu \\
& \beta=288 \mu \\
& \partial=88 \mu
\end{aligned}
$$

\begin{tabular}{|c|c|c|c|}
\hline & PAtte I & Patte II & PAtte III \\
\hline . & & & \\
\hline Rapport: $\frac{\text { tibia }}{\text { fémur }} \cdots \ldots \ldots \ldots \ldots \ldots \ldots$ & 1,16 & 1,37 & 1,52 \\
\hline Rapport: $\frac{\operatorname{tarse} 1}{\operatorname{tarse} 2} \ldots \ldots \ldots \ldots \ldots \ldots$ & 1,78 & 2 & 2 \\
\hline $\begin{array}{l}\text { Le tarse } 1 \text { est par rapport aux deux } \\
\text { tiers du tibia..................... }\end{array}$ & $<$ & $<$ & $<$ \\
\hline Rapport: $\frac{\text { patte (sans coxa ni troch.) }}{\text { longueur de l'aile }} \ldots$ & 1,33 & 1,45 & 1,65 \\
\hline Rapport: $\frac{\text { patte (sans coxa ni troch.) }}{\text { taille de l'insecte }} \ldots$ & 1,01 & 1,14 & 1,25 \\
\hline
\end{tabular}

$$
\frac{\grave{\alpha}}{\bar{x}}=0,24
$$

\section{Pattes}

\section{Armature génitale}

Les deux segments des gonapophyses supérieures (fig. 1) sont très allongés, le segment proximal étant plus long que le segment distal. 
Le segment distal, grêle, à bords parallèles, porte cinq épines courtes, trois à l'extrémité apicale, les deux autres sur le côté interne environ à l'union du tiers inférieur et des deux tiers supérieurs.

Les gonapophyses inférieures sont également très grêles et plus courtes que les segments proximaux des gonapophyses supérieures: elles portent à leur extrémité apicale deux épines très courtes.

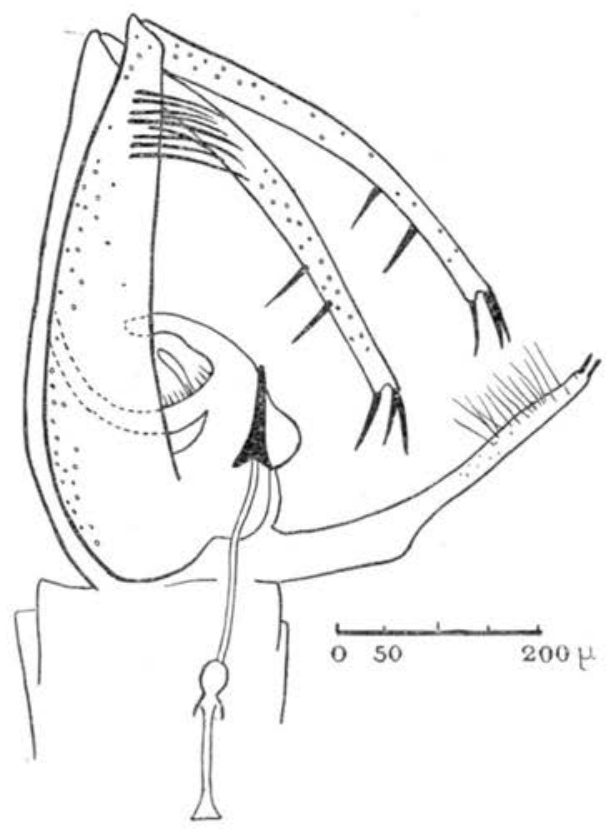

FIG. 1. - Armature génitale mâle de Phlebotomus papatasi (exemplaire de Marseille) (schématique).

Les appendices intermédiaires sont pourvus, en haut, de deux autres appendices secondaires : les frangés et les digitiformes.

Dimensions :

Gonapophyse supérieure : segment proximal ....... $576 \mu$ segment distal ........... $470 \mu$

Gonapophyse inférieure ................. $432 \mu$

Epines des gonapophyses supérieures $\ldots \ldots \ldots \ldots \ldots \ldots .58$ à $72 \mu$

Epines des gonapophyses inférieures ............ $40 \mu$

Appendices intermédiaires .................. $180 \mu$

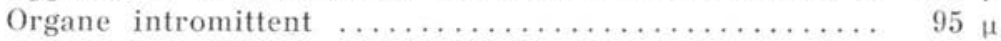

Pompe génitale ...................... $162 \mu$

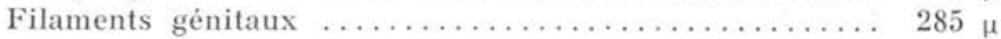


La pompe génitale se trouve dans les deux avant-derniers segments de l'abdomen.

Les divers rapports génitaux sont les suivants :

$$
\begin{aligned}
& \frac{\text { Segment proximal de la gonapophyse supérieure }}{\text { Segment distal de la gonapophyse supérieure }}=1,23 \\
& \frac{\text { Gonapophyse inférieure }}{\text { Segment proximal de la gonapophyse supérieure }}=0,75 \\
& \frac{\text { Segment proximal gonapophyse supérieure }}{\text { Abdomen }}=0,60 \\
& \frac{\text { Longueur des filaments génitaux }}{\text { Longueur de la pompe génitale }}=1,75
\end{aligned}
$$

Femelle

Couleur: jaune pâle.

\section{Dimensions}

A signaler, en ce qui concerne les dimensions, que cet-exemplaire a été traité par la potasse.

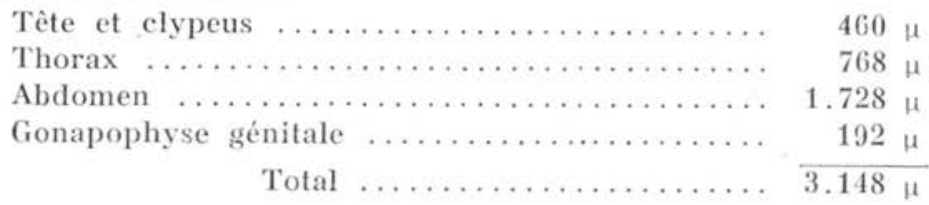

\section{Antennes}

$$
\begin{aligned}
& \mathrm{III}>\mathrm{IV}+\mathrm{V} . \\
& \mathrm{III}<\mathrm{IV}+\mathrm{V}+\mathrm{VI} . \\
& \mathrm{III}<\mathrm{XII} \ldots \mathrm{XVI} . \\
& \mathrm{IV}+\mathrm{V}<\mathrm{XII} \ldots \mathrm{XVI} . \\
& \mathrm{IV}+\mathrm{V}+\mathrm{VI}<\mathrm{XII} . . \mathrm{XVI} .
\end{aligned}
$$

Les épines géniculées, bien développées, sont bigéminées : la formule antennaire est :

$$
\frac{2}{1 I I-X V}
$$

\section{Palpes}

La formule palpale est : 1, 4, 2, 3, 5 .

$$
\begin{array}{ll}
\mathrm{V}<\mathrm{II}+\mathrm{III}+\mathrm{IV} . & \\
\mathrm{V}>\mathrm{III}+\mathrm{IV} . & \mathrm{V} \\
\mathrm{V}=\mathrm{II}+\mathrm{III} . & \\
\mathrm{II}<\mathrm{III} . &
\end{array}
$$




\section{Epipharynx}

La longueur de l'épipharynx est de $334 \mu$.

Le rapport :

$$
\begin{aligned}
& \frac{\text { palpe }}{\text { épipharynx }}=3 \\
& \text { Cavité buccale }
\end{aligned}
$$

Il n'y a pas d'armature buccale.

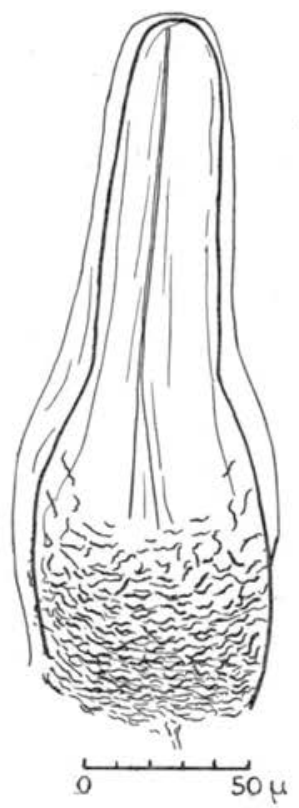

FIG. 2. - Pharynx et armature pharyngienne de Phlebotomus papatasi femelle (exemplaire de Marseille) (schématique).

\section{Pharynx}

Le pharynx est assez élargi à sa partie postérieure. Sa longueur est environ trois fois sa plus grande largeur qui, elle, a des dimensions d'un peu plus du double que la partie rétrécie antérieure. L'armature (fig. 2) donne l'impression d'un réseau ou d'une mosaïque dont la surface antérieure est concave; les denticules ne sont pas distincts.

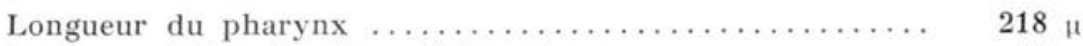

Largeur minima (partie antérieure) $\ldots \ldots \ldots \ldots \ldots \ldots \ldots . \ldots \ldots$

Largeur maxima (partie postérieure) $\ldots \ldots \ldots \ldots \ldots \ldots \ldots . \quad 70 \mu$

\section{Aile}

Le bord postérieur de l'aile est plus arqué que le bord antérieur. La première nervure longitudinale recouvre la branche antérieure de la deuxième sur le quart environ de sa longueur. 
La branche antérieure de la deuxième nervure longitudinale est plus longue que la distance entre les deux fourches : $\alpha>\beta$.

La fourche postérieure de la deuxième nervure longitudinale est plus rapprochée de la base de l'aile que la fourche de la quatrième.

Longueur de l'aile ........................ $2.112 \mu$

Largeur de l'aile (maxima) $\ldots \ldots \ldots \ldots \ldots \ldots \ldots \ldots \ldots \ldots \ldots \ldots \ldots \ldots \ldots \ldots$

$$
\begin{aligned}
& \frac{\text { Longueur de l'aile }}{\text { Largeur de l'aile }}=3.23 \\
& \frac{\text { Taille de l'insecte }}{\text { Longueur de l'aile }}=1,49
\end{aligned}
$$$$
\begin{aligned}
& \alpha=432 \mu \\
& \beta=288 \mu \\
& \delta=105 \mu
\end{aligned}
$$$$
\frac{\alpha}{\beta}=1,50
$$$$
\frac{\delta}{\alpha}=0,24
$$

\begin{tabular}{|c|c|c|c|}
\hline & Patte I & Patte II & Patte III \\
\hline Rapport: $\frac{\text { tibia }}{\text { fémur }} \cdots \ldots \ldots \ldots \ldots \ldots \ldots$ & 1,05 & 1,24 & 1,37 \\
\hline Rapport $: \frac{\operatorname{tarse} 1}{\operatorname{tarse} 2} \ldots \ldots \ldots \ldots \ldots \ldots \ldots$ & 1,80 & 2 & 2,02 \\
\hline 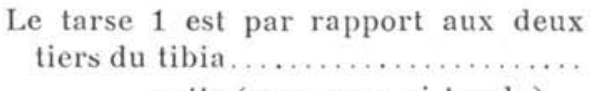 & $<$ & $<$ & $<$ \\
\hline Rapport: $\frac{\text { patte (sans coxa ni troch.) }}{\text { longueur de l'aile }} \ldots$ & $-1,30$ & 1,48 & 1,80 \\
\hline Rapport: $\frac{\text { patte (sans coxa ni troch.) }}{\text { taille de l'insecte }} .$. & 1,04 & 1,18 & 1,42 \\
\hline
\end{tabular}

\section{Pattes}

\section{Spermathèques}

Elles sont situées dans la partie distale du septième segment abdominal (fig. 3). Elles ont un aspect en boudin, tronquées antérieurement, à parois nettement crénelées. L'anneau apical n'est pas plus large que les autres ; le cou très court et la tête élargie sont entourés par une collerette ayant la largeur de l'organe. Les crénulations sont au nombre de 8 à 10 et chaque spermathèque mesure de 45 à $50 \mu$ de long sur 16 à $17 \mu$ de large.

Etant donnés surtout les caractères typiques fournis par l'armature génitale du mâle, d'une part, et, d'autre part, par la morphologie des spermathèques, l'absence d'armature buccale et la disposition de l'armature pharyngienne chez la femelle, le diagnostic de Phlebotomus papatasi s'impose pour ces deux exemplaires. 
Dans un article récent (1), Langeron et Nitzulescu, passant en revue la répartition des phlébotomes en France, à la suite de nouvelles identifications poursuivies sur des exemplaires déjà diagnostiqués, attiraient l'attention sur le fait suivant: le diagnostic de Phlebotomus papatasi fut porté à tort dans la plupart des localités où des phlébotomes avaient été précédemment capturés et décrits.

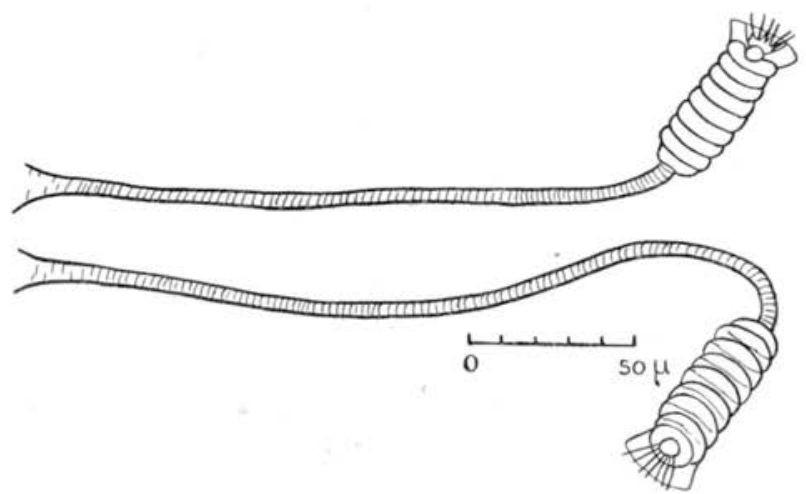

Fig. 3. - Spermathèques de Phlebotomus papatasi (exemplaire de Marseille) (schématique).

Contrairement à l'opinion courante, concluaient-ils, Phlebotomus papatasi, espèce la plus anciennement connue, paraît rare en France : on ne connait, à l'heure actuelle, qu'une seule localité où cette espèce ait été précédemment identifiée de façon certaine et authentique : Montpellier (en réalité localité de Valéry Mayet aux environs de Montpellier).

A cette unique localité, il y a donc lieu d'ajouter Marseille.

\section{RÉSUMÉ}

Deux échantillons de Phlebotomus papatasi Scopoli 1786, un mâle et une femelle ont été identifiés à Marseille, quartier de la Corniche.

Cette localité doit done être considérée comme un des points de la répartition géographique de cette espèce de phlébotome en France continentale.

\section{Laboratoire de parasitologie de l'Ecole d'application du Service de Santé des troupes coloniales.}

(1) M. Langeron et V. Nitzulescu. - Révision des phlébotomes de France. Ann. de Parasitologie, X, 1932, p. 286-294. 